\title{
Interstellar Chemistry: Radiation, Dust and Metals
}

\author{
Marco Spaans ${ }^{1}$ \\ ${ }^{1}$ Kapteyn Astronomical Institute, University of Groningen, \\ P.O. Box 800, 9700 AV, Groningen, the Netherlands \\ email: spaans@astro.rug.nl
}

\begin{abstract}
An overview is given of the chemical processes that occur in primordial systems under the influence of radiation, metal abundances and dust surface reactions. It is found that radiative feedback effects differ for UV and X-ray photons at any metallicity, with molecules surviving quite well under irradiation by X-rays. Starburst and AGN will therefore enjoy quite different cooling abilities for their dense molecular gas. The presence of a cool molecular phase is strongly dependent on metallicity. Strong irradiation by cosmic rays $(>200 \times$ the Milky Way value) forces a large fraction of the $\mathrm{CO}$ gas into neutral carbon. Dust is important for $\mathrm{H}_{2}$ and HD formation, already at metallicities of $10^{-4}-10^{-3}$ solar, for electron abundances below $10^{-3}$.
\end{abstract}

Keywords. astrochemistry, ISM: molecules, dust, early universe

\section{Introduction}

In the study of primordial chemistry, and subsequently the formation of the first stars, it is crucial to understand the ability of interstellar gas to cool through atomic and molecular emissions and to collapse. Furthermore, atomic and molecular species can be used to probe the ambient conditions, such as density and temperature, under which stars form. The basic questions posed by this contribution are: What sets the abundances of atoms and molecules that cool the gas? What is the role of radiation, metallicity and dust in molecule formation?

A number of different chemical processes are relevant to this effect:

Ion-molecule reactions: $\mathrm{A}^{+}+\mathrm{BC} \rightarrow \mathrm{AB}^{+}+\mathrm{C}$;

Neutral-neutral reactions: $\mathrm{A}+\mathrm{BC} \rightarrow \mathrm{AB}+\mathrm{C}$;

Dissociative recombination: $\mathrm{AB}^{+}+\mathrm{e}^{-} \rightarrow \mathrm{A}+\mathrm{B}$;

Radiative recombination: $\mathrm{A}^{+}+\mathrm{e}^{-} \rightarrow \mathrm{A}+h \nu$;

Radiative association: $\mathrm{A}+\mathrm{B} \rightarrow \mathrm{AB}+h \nu$;

Ionization: $\mathrm{A}+\mathrm{CR} / \mathrm{UV} / \mathrm{X}-\mathrm{ray} \rightarrow \mathrm{A}^{+}+\mathrm{e}^{-}$;

Dissociation: $\mathrm{AB}+\mathrm{UV} \rightarrow \mathrm{A}+\mathrm{B}$;

Charge transfer: $\mathrm{A}^{+}+\mathrm{B} \rightarrow \mathrm{A}+\mathrm{B}+$;

Grain surface reactions: Grain $+\mathrm{A}+\mathrm{B} \rightarrow$ Grain $+\mathrm{AB}$.

In any chemical network, the above reactions play an important role. For example, the charge transfer between $\mathrm{H}^{+}$and $\mathrm{O}$, followed by reactions with $\mathrm{H}_{2}$ to $\mathrm{H}_{3} \mathrm{O}^{+}$, and dissociative recombination with $\mathrm{e}^{-}$, leads to species like $\mathrm{OH}$ and $\mathrm{H}_{2} \mathrm{O}$ (following certain branching ratios). Similarly complex routes exist for CO. In any case, many species are typically joined through different chemical routes. Thus, it is not trivial to construct concise chemical networks if one wants to include important molecules such as CO and 


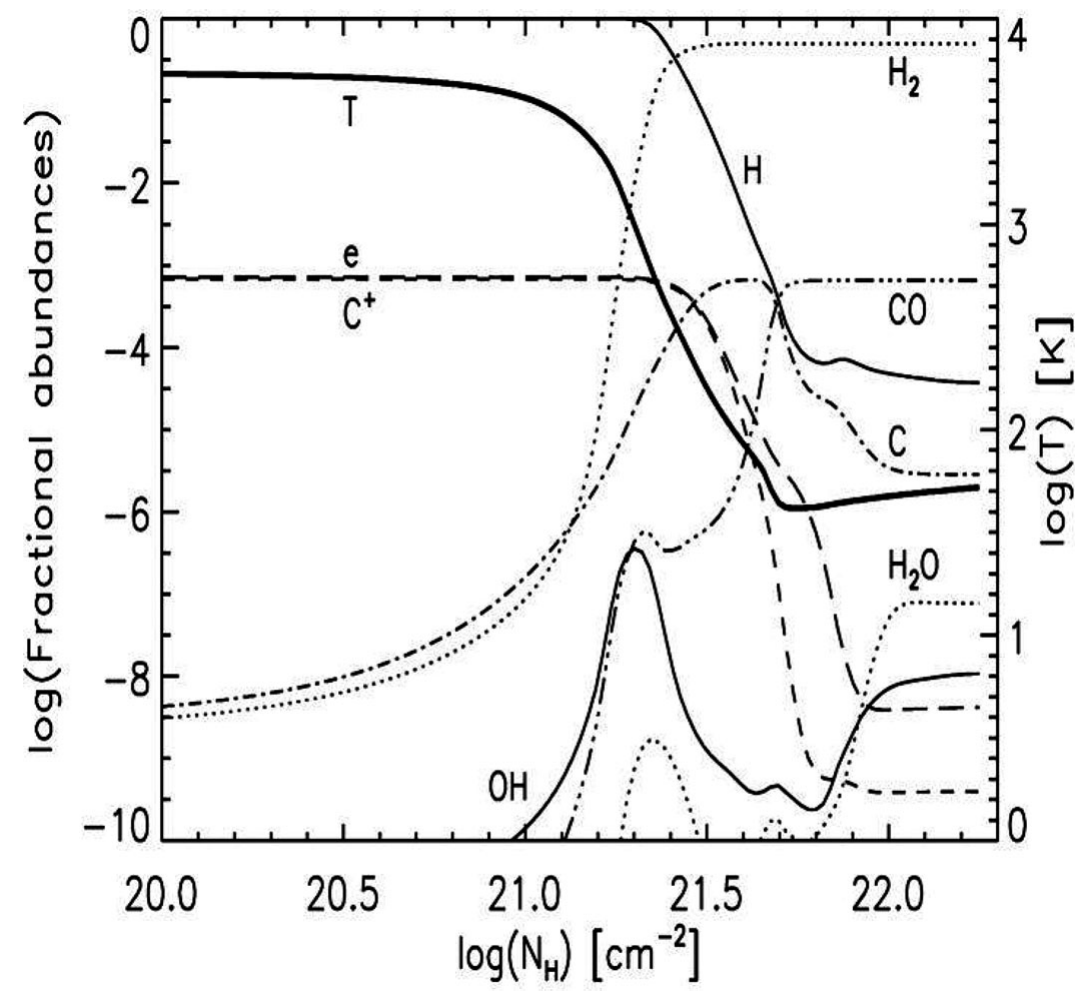

Figure 1. PDR model typical of a modest starburst inside a dwarf galaxy, at a density of $10^{5} \mathrm{~cm}^{-3}$.

$\mathrm{H}_{2} \mathrm{O} \dagger$. Of course, in the limit of low metallicity, chemistry simplifies. Basically, no metals implies no molecules except for $\mathrm{H}_{2}$ and $\mathrm{HD}$ (and a few minor species). Still, even small amounts of metals and dust $\left(\sim 10^{-4}\right.$ solar $)$ can be crucial to the efficient formation of species such as $\mathrm{H}_{2}, \mathrm{HD}, \mathrm{CO}, \mathrm{H}_{2} \mathrm{O}$ and many others, which is the purpose of this contribution.

\section{Radiation}

The impact of radiation is denoted by UV and X-ray dominated regions (PDRs and $\mathrm{XDR}$, respectively). These are regions where photons dominate the thermal and chemical balance of the gas. Examples are O \& B stars (HII regions), active galactic nuclei (AGN), and T Tauri stars. In PDRs, the radiation field comprises photons with energies $6<E<$ $13.6 \mathrm{eV}$. Heating is provided by photo-electric emission from dust grains and cosmic rays, while cooling proceeds through fine-structure emission lines like [OI] 63, $145 \mu \mathrm{m}$ and [CII] $158 \mu \mathrm{m}$ as well as emissions by $\mathrm{H}_{2}, \mathrm{CO}$ and $\mathrm{H}_{2} \mathrm{O}$ rotational and vibrational lines. As a rule of thumb, a $10 \mathrm{eV}$ photon penetrates about $1 / 2$ mag of dust.

In XDRs photon energies $E>0.3 \mathrm{keV}$ are considered. Heating is provided by $\mathrm{X}$-ray photo-ionizations that lead to fast electrons and Coulomb heating as well as $\mathrm{H}$

$\dagger$ It is important to realize that water can be quite an important heating agent in the presence of a warm infrared background, like $T>50 \mathrm{~K}$ dust or a $z>15 \mathrm{CMB}$ (Spaans \& Silk 2000). 


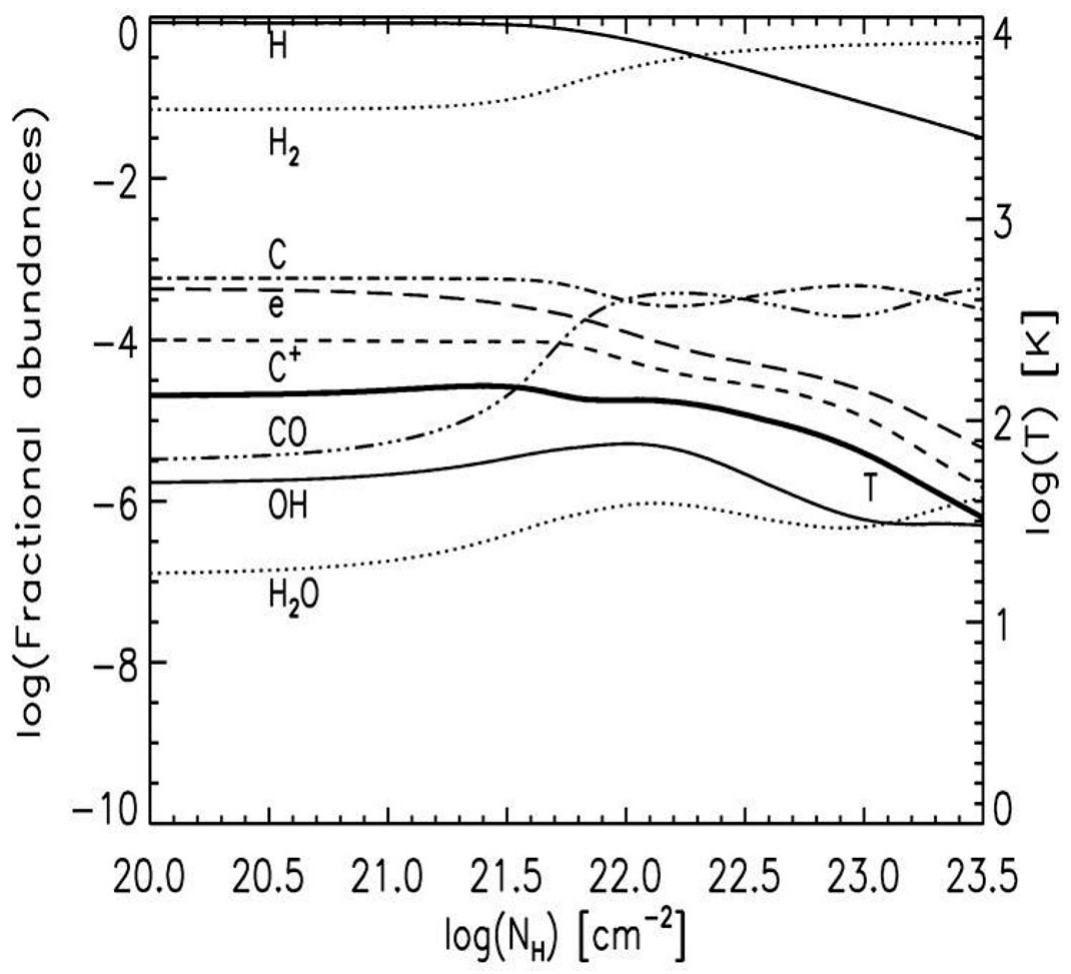

Figure 2. XDR model typical of gas at a few hudred pc from a Seyfert nucleus, at a density of $10^{5} \mathrm{~cm}^{-3}$.

and $\mathrm{H}_{2}$ vibrational excitation followed by UV emission (Ly alpha, Lyman-Werner); $\mathrm{H}_{3}^{+}$ recombination heating can be important as well. Cooling is provided by [FeII] 1.26, $1.64 \mu \mathrm{m}$; [OI] $63 \mu \mathrm{m}$; [CII] 158 and [SiII] $35 \mu \mathrm{m}$ emission lines as well as thermal $\mathrm{H}_{2}$ rotational and vibrational emissions and gas-dust cooling. Typically, a $1 \mathrm{keV}$ photon penetrates $10^{22} \mathrm{~cm}^{-2}$, because cross sections scale as $E^{-(2-3)}$. Figures 1 and 2 show typical examples of a PDR and XDR. Note the fact that molecules have an easier time surviving in an XDR, for the same impinging flux by energy (Meijerink \& Spaans 2005), because molecular photo-dissociation cross sections peak in the UV. Furthermore, the heating efficiciency in XDRs can be $10-50 \%$, while it is at most $1 \%$ in PDRs.

In Figure 3 it is shown that neutral carbon is an excellent mass tracer (as good as $\mathrm{CO})$ under cosmic ray irradiations that exceed Milky Way values by more than factor of 100 (Meijerink et al. 2007). Also note that the collisional coupling between warm gas on cool dust grains can dominate the gas cooling for modest metallicities in PDRs and XDRs.

\section{Metals}

As the metallicity decreases, one finds smaller molecular clouds and the atomic cooling dominates by mass (Bolatto et al. 1999, Roellig et al. 2006). The occurrence of a multi-phase medium (Wolfire et al. 1995) depends strongly on metallicity and pressure 


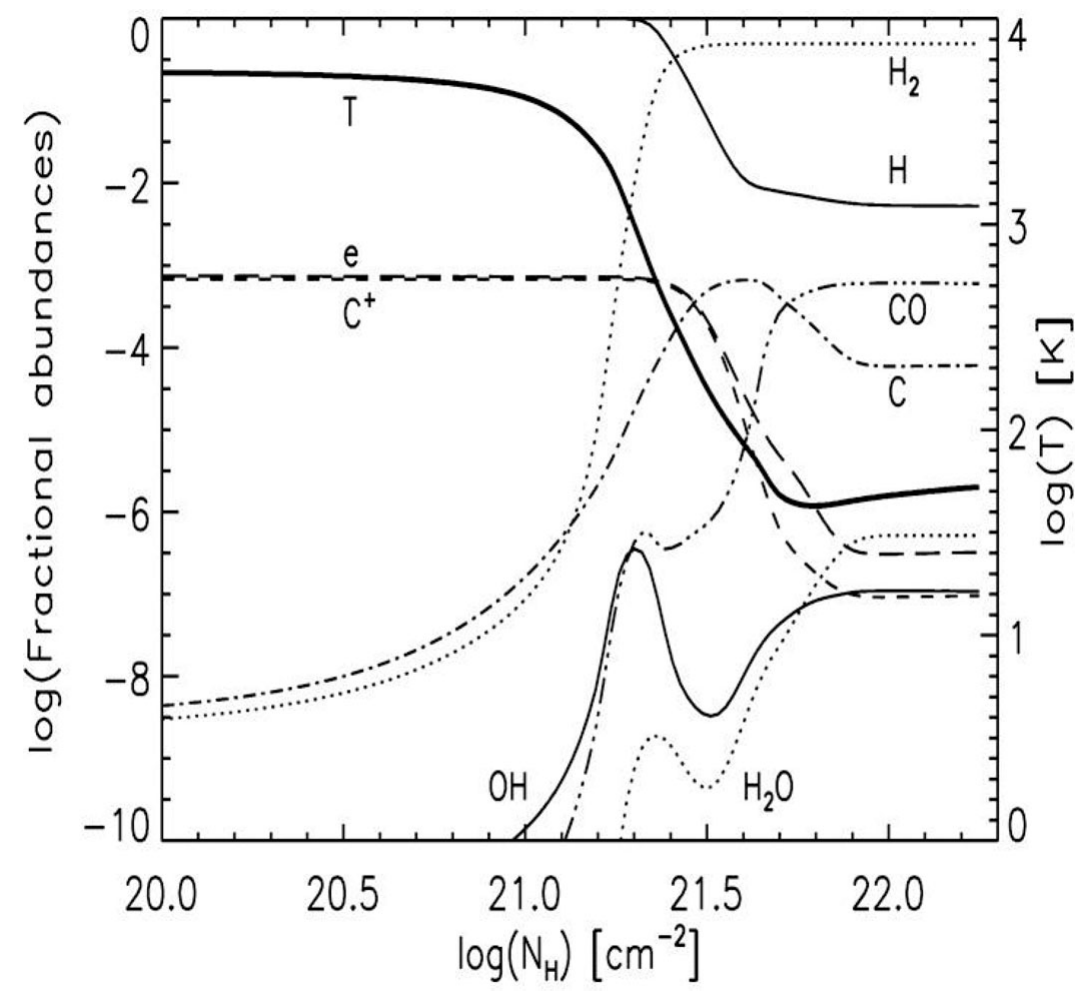

Figure 3. PDR model typical of gas irradiated by cosmic rays from a supernova rate of 2 per year, at a density of $10^{5} \mathrm{~cm}^{-3}$.

(Spaans \& Norman 1997). Figure 4 shows that only a single interstellar phase occurs for metallicities below a percent of solar. At the same time, there is a region between 1 and $10 \%$ of solar metallcity where star formation is most efficient. The reason is that these modest metallicities allow for efficient cooling without any line trapping (optical depth effects).

At metallicities well below $1 \%$ of solar, cooling is dominated by $\mathrm{H}_{2}$ and $\mathrm{HD}$ emissions, which allow cooling down to $\sim 100 \mathrm{~K}$ only. This is illustrated in Figure 5 , where the strengths of the first two pure rotational $\mathrm{H}_{2}$ lines are compared to the $\mathrm{CO}$ line spectral energy distribution, for a system with a $10^{5} \mathrm{M}_{\odot}$ black hole accreting at Eddington. One can clearly see that both low metallicities and strong irradiation favor $\mathrm{H}_{2}$ as the main coolant.

This is pertinent to the study of pop III.1 and III.2 star formation (e.g., Abel et al. 2002, Bromm et al. 2001; and contributions by Schneider, Ferrara and Tan in this volume). The relative contributions of molecular cooling depicted in Figure 5 show that the upcoming ALMA telescope will be able to see primordial systems that are growing a massive black hole, at redshifts of $z=10-20$ (Spaans \& Meijerink 2008).

Metallicity-dependent cooling is quite important for the collapse of gas clouds and the properties of the initial mass function. In particular, LTE effects and line trapping impact the effective equation of state (the thermodynamics) of the gas. This is further 

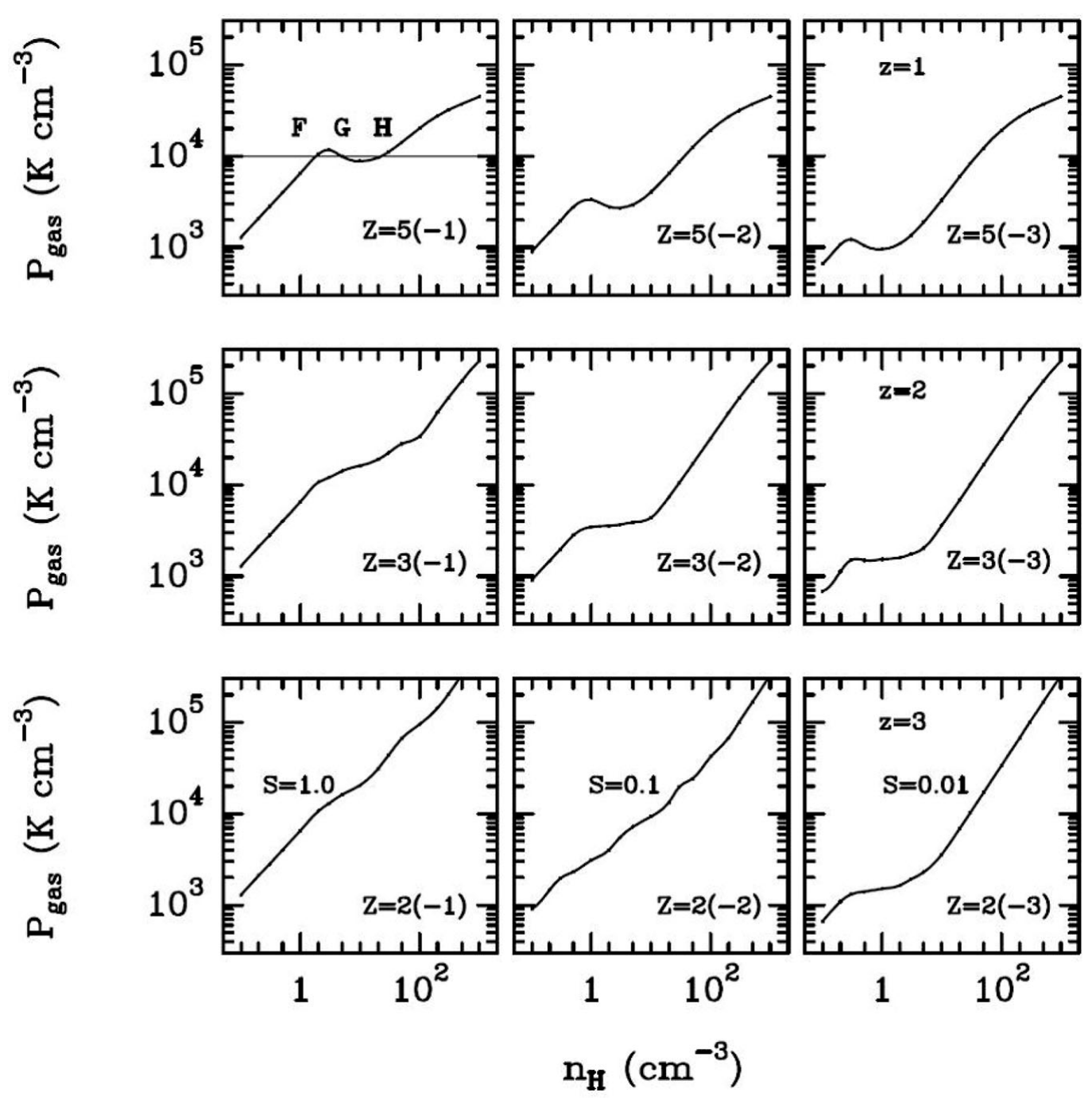

Figure 4. Phase diagrams for interstellar gas as a function of metallicity and background star formation rate.

discussed in detail, using the FLASH code, in the contribution of Hocuk (this volume). He finds that the level of fragmentation is a strong function of rotational energy and metallicity.

Furthermore, metals and molecules other than $\mathrm{H}_{2}$ and $\mathrm{HD}$ impact the formation of structure in collapsing primordial systems. Detailed studies, using the Enzo code, that include a complete gas-phase chemistry and formation of $\mathrm{H}_{2}$ and $\mathrm{HD}$ on dust grains, is presented by Aykutalp (this volume). She finds that pre-enrichment of young galaxies strongly lowers the Jeans mass of the gas clouds they contain. 


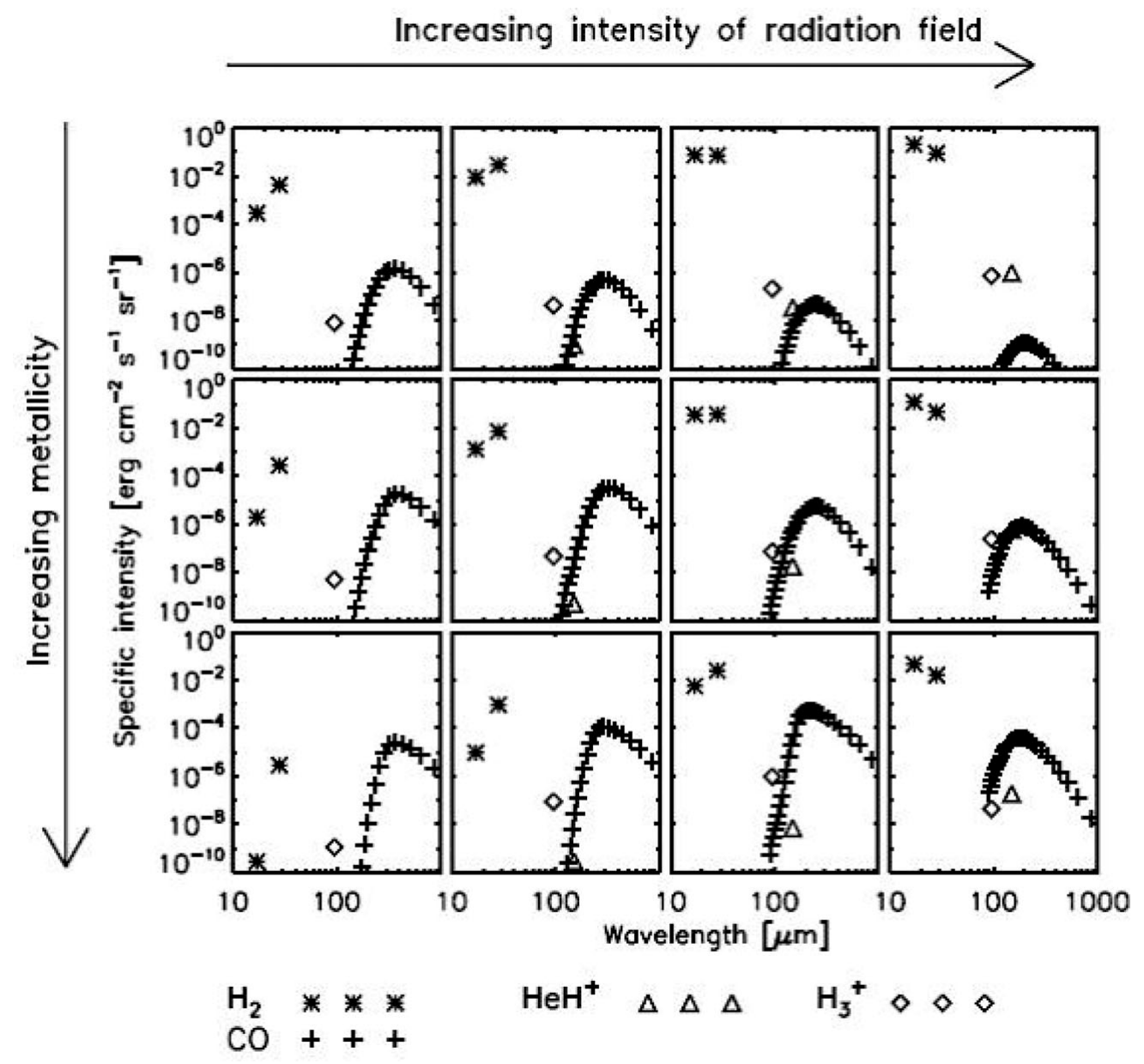

Figure 5. Relative contributions from $\mathrm{CO}$ (metal-rich) and $\mathrm{H}_{2}$ (metal-poor) gas that is irradiated by a hard spectrum of primordial galaxy; as a function of metallicity $\left(10^{-3}\right.$ to $10^{-1} /$ top to bottom) and impinging flux (0.1 to $100 \mathrm{erg} \mathrm{cm}^{-2} \mathrm{~s}^{-1} /$ left to right). All panels are for a density of $10^{5} \mathrm{~cm}^{-3}$.

\section{Dust}

The formation of $\mathrm{H}_{2}$ and $\mathrm{HD}$ on dust grains depends strongly on their surface properties, as indicated in Figure 6. Hydrogen atoms can be weakly bound through van der Waals forces (physi-sorption) or strongly bound through covalent bonds (chemi-sorption). The advantage of the latter bond is that it allows atoms to bind to the surface even for dust temperatures well in excess of $100 \mathrm{~K}$. The hydrogen atoms either thermally hop at high dust temperatures or tunnel at low $(\sim 10 \mathrm{~K})$ temperatures. A comparison with the gas phase formation of $\mathrm{H}_{2}$ through the $\mathrm{H}^{-}$route (see Figure 7 ) indicates that dust processes dominate $\mathrm{H}_{2}$ formation for metallicities $>10^{-3.5}$ solar and electron abundances below $10^{-3}$ (Cazaux \& Spaans 2004).

The formation of HD benefits above $10^{-3}$ solar as well, as long as the gas density is above $10^{4.5} \mathrm{~cm}^{-3}$ and the electron abundance below $10^{-3}$ (Cazaux \& Spaans 


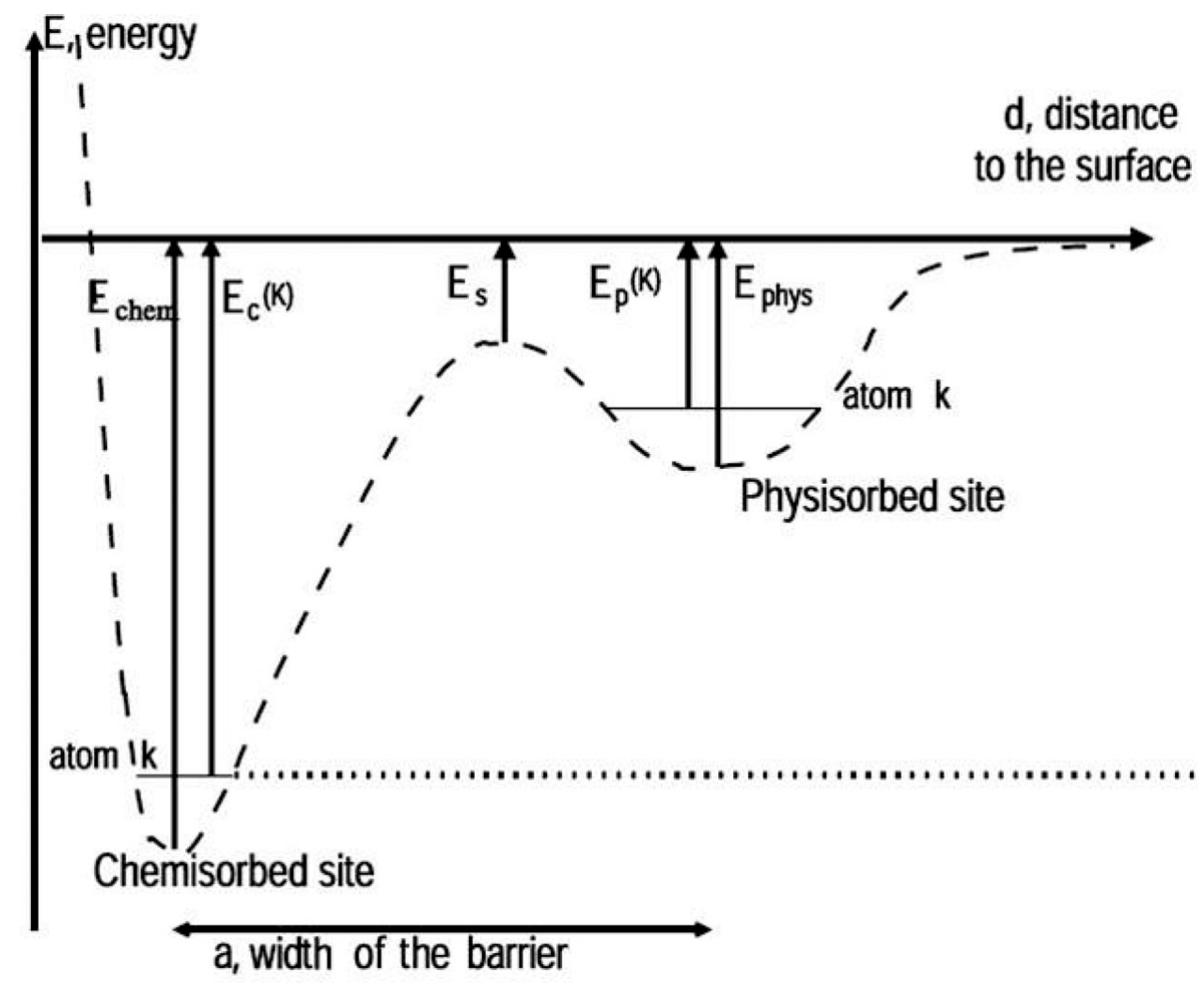

Figure 6. Typical grain surface characterization (Cazaux \& Tielens 2004).

2008 , in preparation). In this, the deuterium atom is more massive that atomic hydrogen, i.e., it is less mobile and more strongly bound to the dust grain (up to higher temperatures).

\section{Conclusions}

Radiative feedback effects differ for UV and X-ray photons at any metallicity, with molecules surviving quite well under irradiation by X-rays. Starburst and AGN will therefore enjoy quite different cooling abilities for their dense molecular gas. The presence of a cool molecular phase is strongly dependent on metallicity. Strong irradiation by cosmic rays $(>200 \times$ the Milky Way value) forces a large fraction of the CO gas into neutral carbon. Dust is important for $\mathrm{H}_{2}$ and $\mathrm{HD}$ formation, already at metallicities of $10^{-4}-10^{-3}$ solar.

Finally, one should always solve the equations of statistical equilibrium to distinguish properly between the excitation, radiation and kinetic temperature of a system. I.e., the thermodynamic floor set by the CMB is only a hard one if the density is high enough (larger than the critical density of a particular transition) to drive collisional de-excitation. 


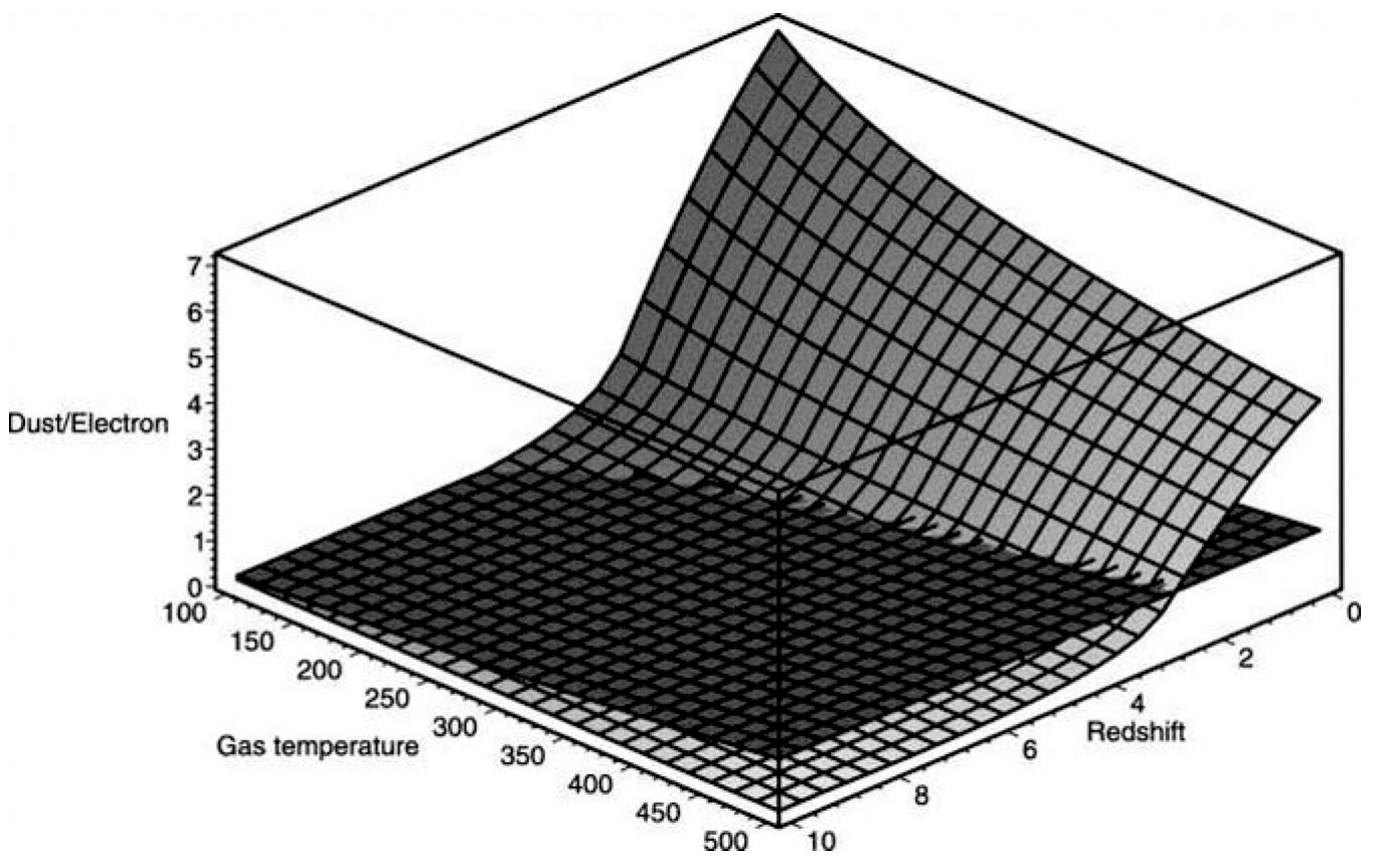

Figure 7. Comparison between the gas phase and dust surface formation routes for $\mathrm{H}_{2}$.

\section{References}

Abel, T., Bryan, G.L., \& Norman, M.L., 2002, Science, 295, 93

Bolatto, A.D., Jackson, J.M. \& Ingalls, J.G., 1999, ApJ, 513, 275

Bromm, V., Ferrara, A., Coppi, P.S., \& Larson, R.B., 2001, MNRAS, 328, 969

Cazaux, S. \& Spaans, M., 2004, ApJ, 611, 40

Cazaux, S. \& Tielens, A.G.G.M., 2004, ApJ, 604, 222

Meijerink, R. \& Spaans, M., 2005, A\&A, 436, 397

Meijerink, R., Spaans, M. \& Israel, F.P., 2007, A\&A, 461, 793

Roellig, M., Ossenkopf, V., Jeyakumar, S., Stutzki, J. \& Sternberg, A., 2006, A\&A, 451, 917

Spaans, M. \& Meijerink, R., 2008, ApJ, 678, L5

Spaans, M. \& Silk, J., 2000, ApJ, 538, 115

Spaans, M. \& Norman, C.A., 1997, ApJ, 483, 87

Wolfire, M.G., Hollenbach, D., McKee, C.F., Tielens, A.G.G.M. \& Bakes, E.L.O., ApJ, 443, 673 